\title{
3D Structures on Relativistic Jets
}

\author{
Philip E. Hardee ${ }^{\mathrm{a}, 1}$, \\ ${ }^{a}$ University of Alabama, Department of Physics \& Astronomy, Tuscaloosa, AL \\ 35487
}

\begin{abstract}
The properties of wave-like helically twisted normal mode structures on steady relativistic jets are summarized. Wave speeds are a function of the wavelength and less than the jet speed. However, normal mode interference can lead to both stationary and superluminal phase effects. A maximum pressure fluctuation criterion suggested by numerical simulations of axisymmetric relativistic jets is used to find the maximum asymmetric jet distortions and velocity fluctuations. Cyclic transverse velocity fluctuation can lead to variation in the flow direction on the order of the relativistic beaming angle. Resulting variation in the Doppler boost factor can lead to significant brightness asymmetries as helical structures twist around the jet beam. Growth of these structures is reduced as the jet density, Lorentz factor or Mach number are increased. Maximum jet distortion is reduced as the Lorentz factor increases and this suggests a reduction in mass entrainment or other non-linear disruptive processes that influence the morphological development of radio sources.
\end{abstract}

\section{Introduction}

Many known or suspected relativistic jets exhibit complex time-dependent structures, e.g., 3C 345 (Zensus, Cohen, \& Unwin 1995) \& M87 (Biretta, Zhou, \& Owen 1995, Biretta, Sparks, \& Macchetto 1999), that are difficult to understand in terms of simple static jet bending and/or acceleration of components along a fixed trajectory. Wiggling or twisted helical structures on multiple length scales provides evidence for wave-like "normal mode" structures along these flows, and precession of the central engine provides a periodic driver that can excite the normal modes on multiple length scales. The amplitudes to which normal mode structures can grow has been investigated for non-relativistic jets (Hardee, Clarke, \& Rosen 1997, Keppens \& Tóth 1999) and for axisymmetric relativistic jets (Hardee et al. 1998) via comparison between theory and numerical simulations. Here we present a prediction of the

1 E-mail: hardee@athena.astr.ua.edu 
maximum amplitudes to which asymmetric flow driven (Kelvin-Helmholtz unstable) structures could grow on hydrodynamic relativistic jets and the effect on observable jet structure and radio source development. Large amplitude normal mode distortions lead to mass entrainment and disruption of highly collimated flow (Rosen et al. 1999, Rosen \& Hardee, these proceedings) and are an important aspect of the morphological development of radio sources.

\section{The Normal Modes}

A perturbation to a cylindrical jet can be considered to consist of Fourier components of the form

$$
f_{1}(r, \phi, z)=f_{1}(r) \exp [i(k z \pm n \phi-\omega t)]
$$

where flow is along the z-axis, and the flow is bounded by $r=R$ in the radial direction. In cylindrical geometry $k$ is the longitudinal wavenumber, $n$ is an integer azimuthal wavenumber, for $n>0$ the angle of the wavevector relative to the flow direction is $\theta=\tan (n / k R)$, and $+n$ and $-n$ refer to wave propagation in the clockwise and counterclockwise sense, respectively, when viewed outwards along the flow direction. The azimuthal wavenumbers $n=0,1,2,3$, etc. correspond to pinching, helical, elliptical, triangular, etc. normal mode distortions of the jet, respectively. For normal mode $n$ the axial wavelength associated with a $360^{\circ}$ helical twist of a wavefront around the jet beam is given by $\lambda_{z}=n \lambda_{n}$ where $\lambda_{n}=2 \pi / k$.

Propagation and growth or damping of the Fourier components is described by a dispersion relation (see Birkinshaw 1991). On the supersonic jet, i.e., $M_{j t} \equiv$ $u / a_{j t}>>1$ and $M_{e x} \equiv u / a_{e x}>>1$ where we define the sound speeds by $a^{2} \equiv$ $\Gamma P /\left\{\rho+[\Gamma /(\Gamma-1)] P / c^{2}\right\}$ and $\Gamma$ is the adiabatic index, each normal mode, $n$, consists of a single "surface" wave and multiple "body" wave solutions to the dispersion relation.

In the low frequency limit all asymmetric $(n>0)$ growing normal mode "surface" wave solutions are given by (e.g. Hardee 1987)

$$
\omega / k u \approx \frac{\gamma^{2} \eta_{\text {rel }}}{1+\gamma^{2} \eta_{\text {rel }}}+i \frac{\gamma \eta_{\text {rel }}^{1 / 2}}{1+\gamma^{2} \eta_{\text {rel }}},
$$

where $\eta_{r e l} \equiv\left(a_{e x} / a_{j t}\right)^{2}$, and $\gamma$ is the Lorentz factor. The growth rate (imaginary part) decreases and wave speed increases as $\gamma^{2} \eta_{\text {rel }}>>1$. In the low frequency limit all "body" wave solutions are given by (see Hardee 1987, Hardee et al. 1998)

$$
k R \approx k_{n m}^{\min } R \equiv \frac{(n+2 m-1 / 2) \pi / 2}{\gamma\left(M_{j t}^{2}-1\right)^{1 / 2}},
$$

where $n$ is the mode number, and $m \geq 1$ is the integer body mode number. In this limit $\left.(\omega / k u)\right|_{\text {real }} \approx 0$ and the body wave solutions are either purely real 
or damped. At higher wavenumbers, $k R \geq k_{n m}^{\ell} R \gtrsim k_{n m}^{\min } R$, the body modes are propagating and growing.

All asymmetric surface and body normal mode waves have a maximum in the growth rate (see Gill 1965, Payne \& Cohn 1985, Hardee 1987, Hardee et al. 1998), at a resonant frequency, wavelength and wave speed:

$$
\begin{gathered}
\omega R / a_{e x} \lesssim \omega_{n m}^{*} R / a_{e x} \equiv(n+2 m+1 / 2) \pi / 2 \\
\lambda \gtrsim \lambda_{n m}^{*} \equiv \frac{2 \pi}{\omega_{n m}^{*} R / u} \frac{\gamma\left[M_{j t}^{2}-u^{2} / c^{2}\right]^{1 / 2}}{\left[M_{e x}^{2}-u^{2} / c^{2}\right]^{1 / 2}+\gamma\left[M_{j t}^{2}-u^{2} / c^{2}\right]^{1 / 2}} R, \\
v_{w} \approx v_{w}^{*} \equiv \frac{\gamma\left[M_{j t}^{2}-u^{2} / c^{2}\right]^{1 / 2}}{\left[M_{e x}{ }^{2}-u^{2} / c^{2}\right]^{1 / 2}+\gamma\left[M_{j t}{ }^{2}-u^{2} / c^{2}\right]^{1 / 2}} u .
\end{gathered}
$$

In the above $m=0$ indicates the surface wave and $m \geq 1$ indicates the body waves. At this resonance the growth rate is proportional to $\left(\gamma M_{j t}\right)^{-1}$ and growth is slowed as $\gamma M_{j t}$ increases.

At high frequencies and short wavelengths the growth rate becomes vanishingly small and the normal modes propagate like sound waves in the jet with

$$
\omega / k u \approx \frac{1-a_{j t} / u}{1-a_{j t} u / c^{2}}
$$

Thus, the normal modes propagate with a wave speed that is a function of the frequency relative to the resonant frequency, and wave speeds are always less than the underlying flow speed.

\section{Normal Mode Structures}

Displacements of jet fluid can be written in the form

$$
\boldsymbol{\xi}(r, \phi, z)=\mathbf{A}\left(r_{0}\right) e^{i \boldsymbol{\Delta}\left(r_{0}\right)} \xi_{r}(R) \exp \left[i\left(k z_{s} \pm n \phi_{s}-\omega t\right)\right],
$$

where $z_{s}$ and $\phi_{s}$ are the axial and azimuthal position at the jet surface, $r_{0}$ is the initial radial position, $\xi_{r}(R)$ is the radial displacement at the jet surface, and the $\mathbf{A}(r) e^{i \boldsymbol{\Delta}(r)}$ are simple relativistic equivalents (Hardee 1999) of equations (A10) in Hardee, Clarke, \& Rosen (1997). The velocity components associated with fluid displacements are given by

$$
\mathbf{v}_{1}(r, \phi, z)=d \boldsymbol{\xi} / d t=-i(\omega-k u) \boldsymbol{\xi}(r, \phi, z) .
$$

The total velocity is $\mathbf{v}(r, \phi, z)=\mathbf{u}+\mathbf{v}_{1}(r, \phi, z)$ where $\mathbf{u}$ is the unperturbed jet velocity along the $z$-axis. The pressure perturbation associated with a fluid displacement can be written in the form (Hardee 2000)

$$
P_{1}(r, \phi, z)=B\left(r_{0}\right) e^{i \Delta\left(r_{0}\right)} \xi_{r}(R) \exp \left[i\left(k z_{s} \pm n \phi_{s}-\omega t\right)\right] .
$$

A maximal pressure fluctuation criterion, $0<P_{0}+P_{1}<2 P_{0}$, found from axisymmetric relativistic jet simulations (Hardee et al. 1998) has been used to compute the maximum expected jet distortions for various Lorentz factors and Mach numbers (Hardee 2000). Figure 1 shows the maximum normal mode 
jet cross section distortions for a jet of Lorentz factor $\gamma=10$ and with $M_{j t} \approx$ $M_{e x} \approx 1.3$ for the helical and elliptical surface wave modes at wavelengths, $\lambda_{h}^{(\imath, j, *)} / R=8.25,3.85,1.88$ and $\lambda_{e}^{(\imath, \jmath, *)} / R=5.54,3.49,1.73$, where the shortest wavelength is at resonance, and for the helical and elliptical first body wave modes at the longest unstable wavelength, $\lambda_{h}^{\ell} / R=3.36$ and $\lambda_{e}^{\ell} / R=2.34$.
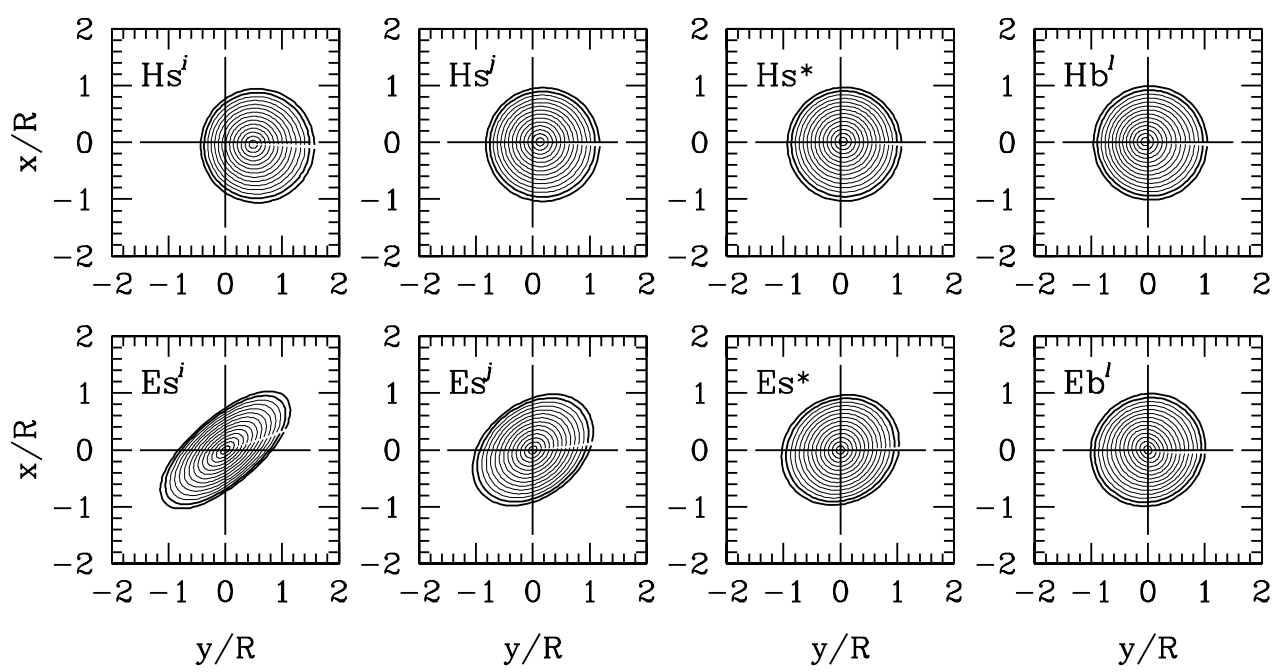

Fig. 1. Maximum cross section distortions for helical and elliptical surface waves at three wavelengths $\left(\lambda^{2}, \lambda^{\jmath}, \lambda^{*}\right)$, and the first body wave at the longest unstable wavelength, $\lambda^{\ell}$.

Jet distortion is a function of the wavelength and scales relative to the resonant wavelength of the appropriate normal mode and not, for example, to the absolute value of the wavelength. High pressure regions are coincident with regions where the displacement surfaces are closer together. The cross sections rotate counterclockwise as the waves propagate outwards along the jet and, in general, high pressure regions precede a trailing low pressure region where the maximum outwards surface displacement occurs. For a given pressure fluctuation, for example at the resonant wavelength, the accompanying jet distortion decreases as the Lorentz factor increases. Thus, in general, the cross sections of jets with large Lorentz factors should be less distorted than those with small Lorentz factors. Still at long wavelengths the helical and elliptical surface modes can lead to significant jet distortion. Other results suggest that most observable (large scale) structure will develop at wavelengths equal to or greater than the resonant wavelengths of the normal modes. Nevertheless, tightly wrapped helical structures are possible on hot relativistic jets with large Lorentz factors because some resonant wavelengths can be less than the jet radius.

The velocity and pressure fluctuations that accompany the distortions can lead to interesting consequences for Doppler boosted emission features associated with asymmetric structures. We can investigate the possible effects on emission features by constructing an "apparent" emissivity, $P^{2} D^{2}$, around 
the displacement surfaces shown in Figure 1 where $P$ is the pressure and $D=\{\gamma[1-(v / c) \cos \theta]\}^{-1}$ is the Doppler boost factor. Let us assume that the initial unperturbed flow angle with respect to the the line of sight is $\theta_{0}=1 / \gamma_{0}=0.1 \mathrm{rad}$, the beaming angle.

Figure 2 gives the values of $P^{2} D^{2}, \gamma, \theta$ and $D$ for the surface modes at the longest wavelength, $\lambda^{2}$, shown in Figure 1. Values are plotted around displacement surfaces with unperturbed radial location of $r_{0} / R=1 / 7,2 / 7, \ldots, 7 / 7$. The azimuthal angle $\phi=0$ lies along the $+y$-axis in Figure 1 and increases in the clockwise sense.
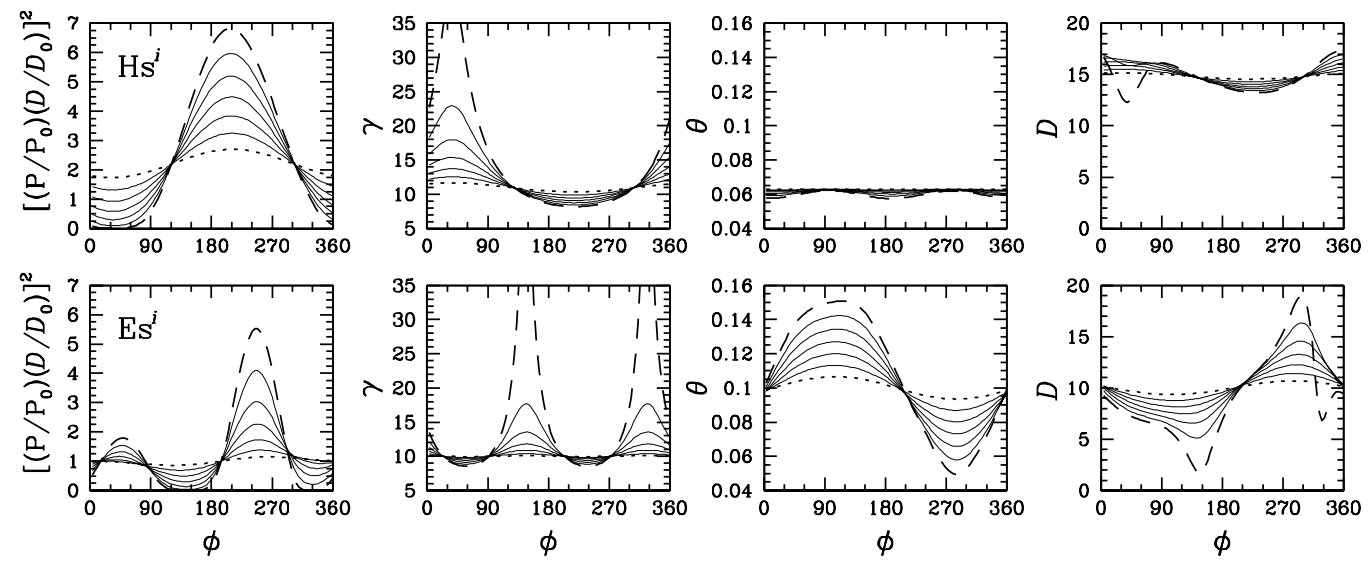

Fig. 2. Emissivity, Lorentz factor, flow angle and Doppler boost factor around the displacement contours for the surface wave at the longest wavelength, $\lambda^{2}$, shown in Figure 1. The dashed (dotted) lines correspond to the surface (center) contours in the jet cross sections, respectively.

For the surface waves the highest pressures and highest "emissivities" occur at the jet surface and for higher order surface modes the distortion amplitude and pressure fluctuation decrease rapidly away from the jet surface. Variation between maximum and minimum apparent emissivity as emission enhanced regions twist around the jet beam, is about a factor of six for the helical mode (not shown) and about a factor of three for the higher order elliptical surface mode, e.g., the difference in emissivity for the two elliptical filaments in Figure 2 .

Figure 3 gives the values of $P^{2} D^{2}, \gamma, \theta$ and $D$ around displacement surfaces for the first body modes at the longest unstable wavelength, $\lambda^{\ell}$. For the body modes the highest pressures and emissivities occur in the jet interior. The variations that occur at the resonant wavelength are similar to those shown in the Figure 3 for the longest unstable wavelength. Surface mode variations at the resonant wavelength are also similar to those shown in Figure 3 although the radial structure is different. 

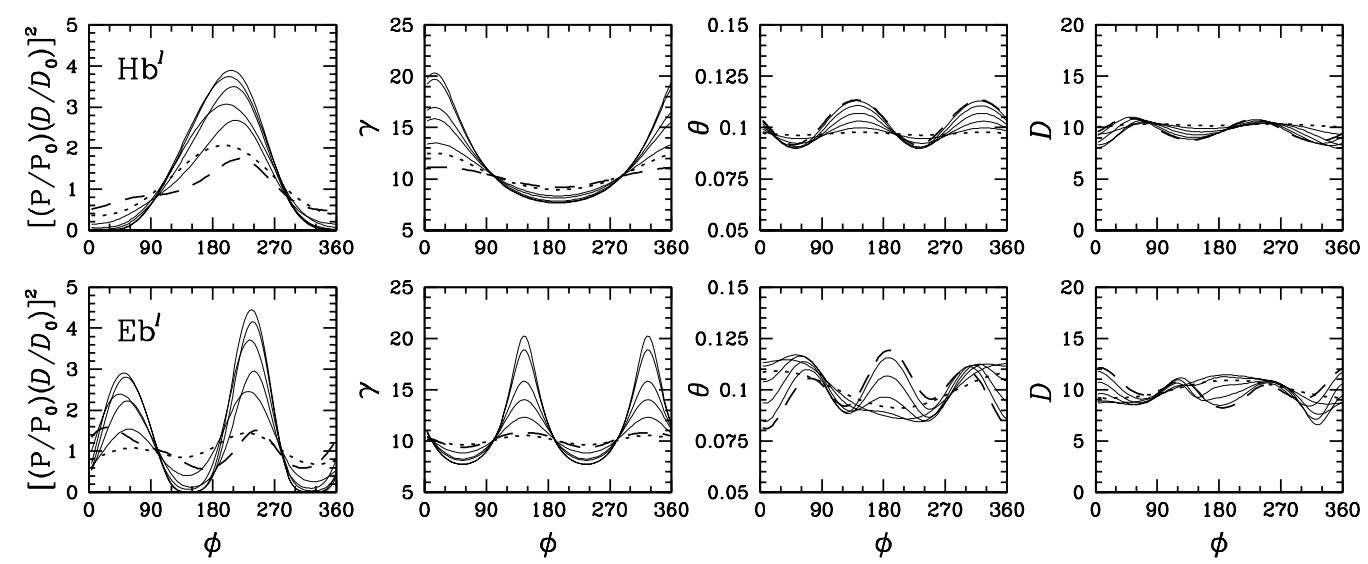

Fig. 3. Like Figure 2 but for the body wave at the longest unstable wavelength shown in Figure 1.

The asymmetric normal modes lead to high pressure regions helically twisted around the jet beam. High pressure regions may be confined close to the jet surface, penetrate deeply into the jet interior or be confined to the jet interior and range from relatively thin sheets and ribbon like to thicker and tube like depending on the mode and wavelength. In general, pressure and emissivity maxima are in phase azimuthally in the jet interior at long wavelengths. At shorter wavelengths the jet center leads the surface and the high pressure region adopts a trailing spiral configuration between jet center and surface. Although axial velocity fluctuation is a small fraction of the relativistic jet speed, significant variation in the Lorentz factor occurs on highly relativistic jets. Even at high Lorentz factor the velocity fluctuations allow for significant angular variation in the flow direction relative to the beaming angle.

\section{Implications}

Jet precession provides a periodic source for the generation of multiple normal mode structures. The interference between normal mode structures of different wavelengths and moving at different wave speeds allows for phase effects producing superluminal and relatively stationary features. Stationary features are created at specific distances from the origin where normal modes of different wavelengths are in phase (Xu, Hardee, \& Stone 2000). Superluminal like motions have not yet been observed in numerical simulations. Non-relativistic numerical simulations have shown resonant pattern motions on the order of the resonant wave speed with motions of localized features within about $\pm 15 \%$ of the average (Hardee 1999, Biretta \& Hardee, unpublished).

High Mach number, jet density and Lorentz factor reduce the growth rate of normal mode structures and high Lorentz factor reduces the maximum amplitude of jet distortions. Nevertheless, significant variation in the Lorentz factor and significant angular variation in the flow direction relative to the 
beaming angle can occur on relativistic jets. Ultimately, if the jet is sufficiently long, large amplitudes at long wavelengths (relative to resonance) can develop and lead to mass entrainment, disruption of highly collimated flow (Rosen et al. 1999, Rosen \& Hardee, these proceedings), and influence the morphological development of radio sources over their lifetime (Eilek, these proceedings).

The jet in M87 provides an illustration of twisted structures on multiple scales that indicate normal mode structures. Radio observations (Owen, Hardee, \& Cornwell 1989) provide strong evidence for a single bright emission filament twisted around the jet beam inside knot D and a fainter twisted filament pair between knots D and F. These features indicate the presence of multiple body and surface modes. Similar structures are evident in VLBI images at parsec scales (Reid et al. 1989). The average motion of well defined structures along the M87 jet is subluminal, $\approx 0.3 c$, and should be taken as indicative of a wave speed and not the speed of the underlying flow. However, motions less than 0.03c and up to about 6c are also observed (Reid et al. 1989, Biretta, Zhou, \& Owen 1995, Junor \& Biretta 1995, Biretta, Sparks, \& Macchetto 1999). Interestingly, the highest observed motions occur for individual bright knots often located in regions with complex structure. At HST-1 (Biretta, Sparks, \& Macchetto 1999) rapidly moving components appear to emerge from a subluminally moving component accompanied by brightness variations up to $\pm 20 \%$. This type of behavior is consistent with interference between different wave modes and line of sight phase effects. In the knot $\mathrm{A}, \mathrm{B}$ and $\mathrm{C}$ complex, the observed bending and oscillation in jet width may be identified with large amplitude helical twisting and elliptical distortion associated with the surface modes. This rapidly growing distortion then leads to mass entrainment and disruption of the highly collimated jet flow.

\section{References}

Biretta, J.A., Sparks, W.B., and Macchetto, F. 1999, ApJ, 520, 621.

Biretta, J. A., Zhou, F., and Owen, F. N. 1995, ApJ, 336, 125.

Birkinshaw, M. 1991, in Beams and Jets in Astrophysics, ed. P.A. Hughes (Cambridge: CUP), 278.

Gill, A.E. 1965, Phys. Fluids, 8, 1428.

Hardee, P.E. 1987, ApJ, 313, 607.

Hardee, P.E., Clarke, D.A., \& Rosen, A. 1997, ApJ, 485, 533.

Hardee, P.E., Rosen, A., Hughes, P.A., \& Duncan, G.C. 1998, ApJ, 500, 599.

Hardee, P.E. 1999, in proceedings of the M87 workshop, eds. H.-J. Röser \& K. Meisenheimer, in press. 
Hardee, P.E. 1999, ApJ, submitted.

Junor, W., and Biretta, J. A. 1995, AJ, 109, 500.

Keppens, R. and Tóth, G. 1999, Phys. of Plasmas, vol.6, no.5, 1461.

Owen, F. N., Hardee, P. E., and Cornwell, T. J. 1989, ApJ, 340, 698.

Payne, D.G. \& Cohn, H. 1985, ApJ, 291, 655.

Reid, M. J., Schmitt, J. H. M. M., Owen, F. N., Booth, R. S., Wilkinson, P. N., Shaffer, D. B., Johnston, K. J., and Hardee, P. E. 1982, ApJ, 263, 615.

Reid, M. J., Biretta, J. A., Junor, W., Spencer, R. E., and Muxlow, T. W. B. 1989, ApJ, 336, 112.

Rosen, A., Hardee, P.E., Clarke, D.A., \& Johnson, A. 1999, ApJ, 510, 136.

Xu, J., Hardee, P.E. and Stone, J.M. 1999, in preparation.

Zensus, J.A., Cohen, M.H., and Unwin, S.C. 1995, ApJ, 443, 35. 